\title{
Essais
}

ESSAIS

Revue interdisciplinaire d'Humanités

Hors-série 4 | 2018

Stanley Kubrick

\section{Dans le labyrinthe du film-cerveau : du concept à l'espace mental}

\section{Pierre Beylot}

\section{(2) OpenEdition}

1 Journals

Édition électronique

URL : http://journals.openedition.org/essais/656

DOI : 10.4000/essais.656

ISSN : 2276-0970

Éditeur

École doctorale Montaigne Humanités

\section{Édition imprimée}

Date de publication : 1 juillet 2018

Pagination : 127-140

ISBN : 979-10-97024-04-8

ISSN : 2417-4211

\section{Référence électronique}

Pierre Beylot, «Dans le labyrinthe du film-cerveau : du concept à l'espace mental », Essais [En ligne], Hors-série 4 | 2018, mis en ligne le 01 décembre 2019, consulté le 16 décembre 2019. URL : http:// journals.openedition.org/essais/656; DOI : 10.4000/essais. 656 


\title{
Dans le labyrinthe du film-cerveau : du concept à l'espace mental
}

\section{Pierre Beylot}

Quel rapport le cinéma entretient-il avec la pensée ? Cette question que soulève Gilles Deleuze dans L'Image-Temps et qu'il considère comme l'un des enjeux de la modernité se pose de manière particulièrement aiguë à propos du cinéma de Kubrick. Chez lui, la pensée ne s'oppose pas au corps, elle s'exprime à travers lui, dans la corporéité de ce que Deleuze appelle le " filmcerveau ", concept qu'il formule à propos de l'œuvre de Kubrick :

\begin{abstract}
"Si l'on considère l'œuvre de Kubrick, on voit à quel point c'est le cerveau qui est mis en scène [...]. C'est que, chez Kubrick, le monde lui-même est un cerveau, il y a identité du cerveau et du monde, tels la grande table circulaire et lumineuse de Docteur Folamour, l'ordinateur géant de 2001, l'odyssée de l'espace, l'hôtel Overlook de Shining. La pierre noire de 2001 préside aussi bien aux états cosmiques qu'aux stades cérébraux : elle est l'âme des trois corps, terre, soleil et lune, mais aussi le germe des trois cerveaux, animal, humain, machinique. Si Kubrick renouvelle le thème du voyage initiatique, c'est parce que tout voyage dans le monde est une exploration du cerveau. Le mondecerveau c'est L'Orange mécanique " ${ }^{1}$.
\end{abstract}

Nous voudrions envisager les différentes facettes de cette métaphore du " film-cerveau » qui articule le dedans et le dehors, l'intériorité de la pensée et l'extériorité des événements, des motifs et des espaces que le film met en scène. En effet, il nous semble que plusieurs strates interprétatives se cristallisent dans cette image : le film-cerveau, cela peut être la pensée en acte du cinéaste ; cela peut être aussi le monde diégétique envisagé comme cerveau ; il peut s'agir enfin de l'espace mental du personnage parcouru selon un axe qui relie l'œil et le cerveau. La première strate est celle du cerveau de Kubrick lui-même, cinéaste souvent perçu comme " conceptuel ", à la fois dans sa manière de concevoir ses films que dans sa propension à soumettre la matière narrative à un concept ou à une hypothèse intellectuelle. La structure de ses films porte la marque de cette prééminence de la pensée sur le récit : une

1 Gilles Deleuze, L'Image-Temps, Paris, Éd. de Minuit, 1985, p. 267-268. 
armature conceptuelle s'y dessine, souvent de manière assez appuyée, elle enveloppe et circonscrit la pensée du film, elle lui donne forme et structure et encadre le récit. La deuxième strate est celle du monde fictionnel envisagé comme monde-cerveau : il ne s'agit plus d'un cadre, mais d'une relation, d'une interface conceptuelle. Celle-ci se traduit par l'émergence de figures où se cristallise la relation entre espace mental et espace physique. Un processus d'échange s'élabore entre le fictionnel et le notionnel : des objets, des lieux, des dispositifs - monolithe, labyrinthe, huis clos... - y fonctionnent à la fois en tant que motifs diégétiques et figures de pensée. Enfin, la troisième strate est celle où s'affirme plus particulièrement la corporéité du film-cerveau, celle de la pensée, souvent tourmentée des personnages. Il ne s'agit pas ici d'une pensée abstraite, mais d'un lien étroit qui s'instaure entre le penser et le voir, l'œil et le cerveau. Chez Kubrick, l'exploration mentale est envisagée dans sa dimension problématique, dans ses apories. En effet, son cinéma donne à voir des univers mentaux obsessionnels et traumatiques et souligne en même temps les limites de cette exploration psychique. Nous allons donc pénétrer dans le labyrinthe du film-cerveau - où parfois Kubrick tente de nous égarer - en privilégiant une décennie élargie, celle des quatre longs-métrages des années 1970 allant de 2001 (1968) à Shining (1980) en passant par Orange mécanique (1971) et Barry Lyndon (1975), car cette période très dense de l'activité créatrice de Kubrick offre un éventail très large et diversifié d'interrelations entre le film et la pensée.

\section{Armature conceptuelle et exosquelette narratif}

On pourrait envisager cette relation entre la pensée et le film sous l'angle du processus créatif : l'univers mental que le film donne à voir, c'est d'abord celui de Kubrick lui-même. D'après les témoignages recueillis dans le documentaire de Jan Harlan, A Life in Pictures (2001), Kubrick était un créateur désireux de maîtriser complètement toutes les étapes de sa création. Steven Spielberg indique ainsi que Kubrick " commençait tous ses films de façon très conceptuelle " et le décrit comme un "illustrateur conceptuel de la condition humaine ». Alan Parker, pour sa part, souligne son contrôle sur le film qui devait apparaître comme la traduction exacte de sa pensée sur l'écran. Mais notre propos porte moins sur la genèse de la création kubrickienne que sur l'univers de pensée qu'elle génère. À cet égard, il faut se demander dans quelle mesure le conceptuel gouverne l'expansion du monde fictionnel. La pensée l'emporte-t-elle chez Kubrick sur le récit? Sans enfermer l'auteur de Shining et de 2001 dans une alternative manichéenne - cinéaste conceptuel versus raconteur d'histoires - il semble que l'imaginaire kubrickien soit traversé par une tension entre ces deux pôles : l'investigation philosophique d'un côté, l'invention de mondes fictionnels de l'autre. Certes, les 
films de Kubrick ne sont pas des films à thèse, ils ne défendent aucune vérité positive, ils ne prétendent véhiculer aucun message, ils développent plutôt une hypothèse dont la signification reste profondément problématique, telle que : « comment associer voyage interstellaire et méditation sur l'existence humaine ?" ou " comment le hasard conduit-il un individu de la richesse à la chute ? ". Nous prenons délibérément deux exemples diamétralement opposés, l'un 2001, affirmant ostensiblement sa dimension spéculative, l'autre Barry Lyndon, plus riche en péripéties narratives, voire plus anecdotique. Mais, dans un cas comme dans l'autre, on a affaire à un film-cerveau que l'on pourrait aussi qualifier de "film-concept ", à condition de considérer que le concept a chez Kubrick une dimension problématique plus que théorématique, pour reprendre l'opposition opérée par Deleuze entre le théorème et le problème ${ }^{2}$. Ainsi, le "film-concept " met-il à l'épreuve une hypothèse qui génère une construction intellectuelle autant qu'une matrice narrative, un univers mental autant qu'une diégèse autonome.

L'hypothèse sur laquelle se fonde le film y imprime sa marque en traçant un cadre - argumentatif aussi bien que narratif - qui charpente fortement le film par des annonces, des cartons, des voix over et des musiques qui forment un discours d'escorte et orientent la lecture du spectateur. C'est là un premier versant du film-cerveau : celui qui consiste à bâtir une armature conceptuelle, à cartographier par avance les parcours interprétatifs en posant des balises aisément repérables. On verra plus loin que cette grille interprétative est en partie un faux-semblant car le film-cerveau peut nous égarer autant que nous guider dans le dédale des connexions logiques qu'il établit. Pour caractériser ce premier mouvement, celui d'une structuration conceptuelle du film, on peut se référer à la notion d' " exosquelette narratif " proposée par Michel Chion ${ }^{3}$. Le terme est usité dans les sciences biologiques et en architecture et désigne le squelette externe de certaines espèces (la carapace des mollusques ou des insectes) ou, par métaphore, l'armature de certains bâtiments (les tubulures de Beaubourg, le treillis métallique du Nid d'oiseau de Pékin).

Dans les films de Kubrick, l'exosquelette repose sur des effets très ostensibles : chapitrage, musiques extradiégétiques, voix over - celle-ci n'étant présente dans notre corpus que dans Orange mécanique et Barry Lyndon. On pourrait penser que la fonction de cet exosquelette est d'abord une fonction d'élaboration et de balisage narratifs : les cartons donnent des repères, par exemple, dans 2001, la mission Jupiter a lieu dix-huit mois après la mission sur la lune (Figure 1) ou entretiennent la tension dramatique (l'entracte intervient à un moment d'acmé narrative après que $\mathrm{Hal}$ a lu sur les lèvres des deux astronautes). Mais ces jalons échappent en grande partie à la chrono-

3 Stanley Kubrick, L’Humain ni plus, ni moins, Paris, Éd. Cahiers du Cinéma, 2004, p. 44-49. 
logie d'un récit : le point de départ ("l'aube de l'humanité ») renvoie à une période difficilement situable et qui a pu s'étaler sur des millénaires (même si elle ne représente que dix-sept minutes de film) et le point d'arrivée ("Jupiter et au-delà de l'infini ") ne désigne plus une planète ni une temporalité identifiables, mais un point de bascule dans une autre dimension, cosmique et métaphysique, celle de l'abstraction temporelle où apparaît le foetus astral. Le chapitrage et les cartons sont donc en partie des leurres : ils semblent des ponctuations narratives, mais jouent en fait le rôle de jalons conceptuels sans que l'on puisse jamais savoir avec certitude en quoi consiste la démonstration.

Dans 2001 comme dans les autres films de notre corpus, tout un travail de distanciation s'opère par rapport à l'exosquelette narrativo-conceptuel que le film élabore, comme si Kubrick s'ingéniait à ruiner l'édifice qu'il est en train de bâtir. L'ironie est l'un de ces effets de distanciation : ainsi, dans Barry Lyndon, les cartons sont utilisés sur le mode du pastiche, reproduisant le style et la graphie du roman picaresque des XVIII ${ }^{e}$ et XIX ${ }^{e}$ siècles et venant annuler, dans le carton final, l'enjeu narratif des intrigues qui agitent des personnages, puissants ou misérables, mais « tous égaux maintenant » dans la mort (Figure 2)... Un autre effet distanciant est l'arbitraire des repères qui forment l'exosquelette narratif. Dans Shining, notamment, la succession des indications temporelles, de plus en plus rapprochées les unes des autres, crée une sorte de pyramide dramatique, un effet compte-à-rebours qui culmine au climax de "4pm " (Figure 3). Mais ces indications ne s'inscrivent pas, là non plus, dans une chronologie, un calendrier de l'action clairement établi, les jours de la semaine semblent interchangeables et leur succession crée une temporalité circulaire et paradoxale. L'exosquelette constitue ainsi une mise en abyme de la structure d'ensemble du récit où les événements dramatiques de 1980 reproduisent ceux des années 1920, comme le révèle le zoom final sur la photographie du bal du 4 juillet 1921 où apparaît la figure fantomatique de Jack Torrance.

La distanciation peut également provenir de l'éclectisme des accompagnements musicaux : le choix d'une illustration musicale est un choix fortement structurant dans l'esthétique de Kubrick ${ }^{4}$. On peut y voir à la fois une des dimensions de l'exosquelette narratif qui oriente la lecture de l'image - que serait 2001 sans Ainsi parlait Zarathoustra et Le Beau Danube bleu? Que serait Orange mécanique sans la Neuvième symphonie de Beethoven ou Barry Lyndon sans la Sarabande de Haendel et le Trio de Schubert ? - mais aussi un élément de déstabilisation du spectateur. Celui-ci est confronté à un patchwork musical où les références les plus hétéroclites se combinent, de la musique symphonique à la comédie musicale, du rock à la musique électronique, comme en témoigne le générique final d'Orange mécanique aux couleurs saturées et violemment contrastées (Figure 4). La musique opère donc à la fois un effet de construction 
et de déconstruction. Le déphasage entre la parole et l'image que l'on pourrait appeler " effet d'hétérochronie " est un autre effet de distanciation pratiqué par Kubrick : la voix over hétérodiégétique, dans Barry Lyndon, anticipe sur ce que montre l'image (par exemple, la mort du comte Lyndon) ou adopte une position surplombante (" combien différent aurait été le sort de Barry s'il ne s'était pas épris de Nora "). Elle participe à l'élaboration d'une armature narrativo-conceptuelle - souvent plus un commentaire des événements qu'une ponctuation narrative - mais qui est souvent en décalage avec les images, d'où un effet de déséquilibre et d'incertitude quant au sens à attribuer au récit.

Toutes ces observations rejoignent l'analyse de Michel Chion qui souligne " la tendance à dé-fusionner les éléments " (parole, image, musique) créant une "fragilisation du récit ». L'exosquelette produit alors " une superposition flottante d'armatures narratives moins contradictoires qu'indépendantes [qui] a pour résultat de fragiliser l'histoire, de donner un sentiment de mystère et de précarité $"^{5}$.

\section{À l'intérieur du film-cerveau : l'interface conceptuelle}

Venons-en maintenant à la construction du monde fictionnel comme un monde-cerveau, autrement dit à ce que nous avons appelé l'interface conceptuelle, la circulation entre espaces mentaux et espaces physiques au travers de lieux ou de figures symboliques. L'un des lieux les plus emblématiques de cette circulation est sans doute le jardin-labyrinthe de Shining (Figures 5, 6 et 7) : c'est d'abord un espace physique, un lieu de déambulation un peu inquiétant de Danny et Wendy dans le premier tiers du film et un lieu terrifiant de poursuite dans le finale ; mais aussi un espace de représentation : la maquette que surplombe littéralement Jack Torrance ; et enfin, un espace symbolique cadré dans une plongée presque verticale qui en fait un lieu abstrait, réactivant la figure mythologique du labyrinthe antique - celui du Minotaure dont Jack est un avatar-, une métaphore du récit et de ses pièges ainsi que de l'espace mental tortueux et torturé des personnages. Le labyrinthe est donc à la fois un lieu physique, matériel, où un certain nombre de péripéties se déroulent, mais aussi un motif visuel qui tend vers l'abstraction.

De manière comparable dans 2001, le monolithe est un objet physique que l'on perçoit par la vue et par l'ouïe (son sifflement strident assourdit les astronautes de la base lunaire), mais c'est aussi une figure symbolique dont la signification reste profondément énigmatique (Figures 8, 9 et 10). Michel Chion a répertorié quelques-unes des interprétations possibles de cette figure :

- Le monolithe est un symbole anthropologique à la manière des pierres érigées de Stonehenge. 
- Le monolithe est totem, phallique.

- Le monolithe est l'anti-monument, l'anti-sépulture.

- Le monolithe, horizontal ou vertical, est symbole de l'abstraction.

- Le monolithe est l'écrit (le livre fermé) en tant qu'il se tait (Hal parle).

- Le monolithe est là et puis "plus là " (cf. Lacan).

- Le monolithe est le Pèré ${ }^{6}$.

Le labyrinthe, le monolithe ou encore la chambre Louis XVI d'" Au-delà de l'infini ", sont des opérateurs d'abstraction qui convertissent les motifs et les lieux fictionnels en espaces symboliques sans opérer une clôture du sens, car la chaîne des interprétations de chacune de ces figures reste infinie. Le dernier chapitre de 2001 témoigne bien de ce glissement vers une symbolisation ouverte à une multiplicité de possibles interprétatifs : la chambre Louis XVI y apparait d'abord dans le cadre ovale de la capsule spatiale avant de s'émanciper de cette référence à l'univers de la science-fiction et de se présenter clairement comme le terme symbolique de l'existence humaine. On semble donc sortir du récit très codé de $\mathrm{SF}$ pour emprunter la voie beaucoup plus abstraite et énigmatique d'une méditation métaphysique, mais il faut bien garder à l'esprit que chez Kubrick coexistent toujours ambition conceptuelle et sens de la dérision qui se manifeste ici par le kitsch du décor d'un blanc immaculé de la chambre Louis XVI qui crée un effet de distanciation ironique.

Deux autres phénomènes témoignent de cette conversion de l'espace fictionnel en un espace symbolique focalisé autour des processus mentaux des personnages : le premier est l'intériorisation de l'action qui se manifeste notamment par la récurrence de la figure du huis-clos. Les vaisseaux spatiaux hermétiquement clos de 2001, les intérieurs éclairés à la bougie de Barry Lyndon, les espaces spectacularisés de violence ou d'enfermement dans Orange mécanique, le labyrinthe et les couloirs de l'hôtel Overlook dans Shining sont autant de dispositifs de restriction de l'espace diégétique qui enferment les personnages dans des espaces de socialisation très restreints, voire dans une solitude presque totale. À des milliers de kilomètres de la terre, au fond d'une prison ou dans un hôtel perdu dans les Rocheuses, les personnages sont coupés du monde et cet isolement entraîne des actions plus intériorisées dans lequel le personnage est d'abord confronté à lui-même et à ses fantasmes violents ou mortiferes. La clôture de l'espace fictionnel tend à symboliser la clôture de l'espace mental des personnages, même lorsque ces derniers se trouvent au sein d'un groupe, comme on peut le voir dans Orange mécanique dans le Korova Milkbar où Alex DeLarge siège au milieu de ses droogies ou dans la petite cuisine étriquée aux couleurs criardes des parents d'Alex dans le même film (Figures 11 et 12).

Le deuxième procédé qui tire l'espace fictionnel vers l'abstraction consiste à traiter les espaces extérieurs comme des décors, et non pas comme des espaces réalistes qui permettraient une immersion illusionniste dans un 
univers transparent. Les effets de rimes visuelles, jouant sur la répétition et la variation autour de certains motifs, contribuent à cette stylisation des espaces fictionnels qui tendent vers la déréalisation et se chargent d'une dimension symbolique. Ainsi, dans Shining, les vues extérieures de l'hôtel Overlook, de plus en plus enseveli sous la neige, ponctuent le déroulement de l'action et effacent progressivement le décor environnant : plus on avance dans le film, plus s'impose une seule réalité tangible, celle de l'hôtel qui n'est plus un lieu matériel, identifiable géographiquement, mais un lieu symbolique où se déchaînent les fantasmes meurtriers de Jack Torrance. La tendance à traiter la réalité diégétique comme un simulacre apparait d'une autre manière dans Barry Lyndon, où se produit un effet " carte postale " avec les plans de châteaux qui rythment le parcours de Barry : le récit picaresque devient une sorte de toile de fond devant laquelle se déploie une action dominée par l'idée fixe du protagoniste, celle d'aller jusqu'au bout d'une ascension sociale qui le conduira à d'amères désillusions et à une fin misérable.

\section{L'axe œil-cerveau : exploration mentale et opacité}

Nous avons décrit les espaces kubrickiens comme des espaces de circulation symbolique où le monde fictionnel renvoie comme en miroir à l'espace mental, mais nous avons pour l'instant laissé de côté un des vecteurs de cette circulation que l'on pourrait appeler "l'axe œil-cerveau ", où se manifeste de la manière la plus profonde la relation entre le corps et la pensée. C'est au travers de l'organe du regard - l'œil - que s'opère cette relation envisagée par Kubrick comme problématique, car l'œil peut donner accès à l'univers mental du personnage, souvent de manière violente, forcée, mais il peut aussi bloquer tout accès à son intériorité, littéralement faire écran. On peut donc envisager cet axe " œil-cerveau » selon deux modalités opposées : celle de l'exploration mentale ou celle de l'opacité.

Avec l'axe œil-cerveau, on n'a plus affaire à des figures symboliques inscrites dans la matérialité d'un objet ou d'un décor (monolithe, labyrinthe), mais d'une figure profondément anthropomorphique qui est celle de l'œil. Le traitement particulier de ce motif dans le cinéma de Kubrick consiste souvent à l'associer à un contexte de violence, d'aliénation, de dépossession comme l'illustre par excellence le «traitement Ludovico» dans Orange mécanique (Figure 13). La vision optique en tant que perception sensorielle - les images de violence et de sexe étant associées ironiquement à la musique de «Ludwig van » - conduit ici à un formatage psychique qui transforme de fond en comble la personnalité du malheureux Alex DeLarge soumis à cette expérimentation.

La vision peut être aussi associée à une autre forme d'expérience-limite, celle de la traversée de «l'au-delà de l'infini » dans 2001 où l'image de l'œil de l'astronaute, soumis à différentes sortes de déformations chromatiques, s'inscrit dans un paysage de formes abstraites et colorées, un univers psychédélique 
dont on ne sait s'il est un espace cosmique ou un espace purement mental. L'œil de Dave est ainsi détaché de son corps, passant dans une autre dimension, dans un au-delà de la vision. Dans le même film, Kubrick joue également sur les limites de la vision et le rapport problématique oeil-cerveau en reproduisant le regard machinique, "l'œil-caméra » en fish-eye de l'ordinateur Hal, d'autant plus redoutable qu'il s'agit d'un oil panoptique, sans lieu assignable, sans corps, mais doté d'un cerveau surpuissant et de capacités perceptives hors du commun comme celle de lire sur les lèvres des astronautes (Figure 14).

L'axe œil-cerveau est également au centre de Shining où s'opère une sorte de court-circuit entre vision optique et vision mentale : le regard fixe, exorbité de Danny, de Jack, de Hallorann et de Wendy face aux hallucinations sanglantes qui les habitent est un regard qui refuse de voir et qui va au-delà de la vision en tant qu'expérience perceptive. Il fait de la vision une expérience traumatique que le personnage ne maîtrise pas et qui nous donne accès à un espace mental dépersonnalisé, puisque partagé par plusieurs personnages de la diégèse : c'est le principe du "shining" comme vision extra-sensorielle (Figure 15).

L'axe œil-cerveau a un premier versant que l'ont vient d'évoquer où l'on a une accessibilité immédiate à l'intériorité tourmentée du personnage. L'autre versant est au contraire celui de l'opacité où l'œil de la caméra ne saisit rien de ses pensées et donne au contraire à voir un visage impénétrable, souvent filmé par de longs zooms arrière. Tous les films de notre corpus offrent de nombreuses occurrences de cet autre traitement de "l'axe oil-cerveau " : c'est, par exemple, le visage énigmatique de Lady Lyndon, perdue dans ses pensées d'épouse délaissée, ou bien le travelling arrière sur le groupe figé d'Alex et de ses droogies au Korova Milkbar, ou encore le zoom avant sur le rictus inquiétant de Jack Torrance, habité de pensées que l'on suppose mortifères mais qu'il est impossible d'identifier précisément. Tous ces moments constituent des stases narratives où le récit est mis en suspens ou entre parenthèses : le seul mouvement est celui de la caméra qui explore le visage mutique des personnages selon une esthétique marquée par une intertextualité plus picturale que cinématographique, entre peinture intimiste pour Barry Lyndon et extravagance kitsch pour Orange mécanique.

Pour conclure, nous voudrions revenir sur la tension, voire la contradiction, entre pensée et récit sur lesquelles nous commencions cet article en retenant deux points en particulier. D'une part, rappelons les trois mouvements qui permettent à cette tension de se déployer au sein de la " noosphère $^{7}$ " kubrickienne : celle d'une armature narrative et conceptuelle qui

7 La notion de "noosphère " a été introduite par Teilhard de Chardin en 1925 et reprise par le philosophe russe Vladimir Vernadsky pour désigner l'univers de la pensée comme système clos. C'est moins la dimension totalisante de la noosphère que nous retenons ici que sa dimension dynamique : chez Kubrick, la sphère de la pensée est un lieu de tensions et de conflits, c'est, selon les mots de Deleuze, «le circuit dans lequel [les pensées] entrent avec l'image-mouvement, 
encadre le récit mais produit aussi bien des effets de déphasage que de structuration ; celle du jeu d'écho et de réflexivité entre intériorité et extériorité, entre espaces fictionnels et espaces mentaux, entre motifs visuels et figures symboliques ; celle, enfin, de la relation problématique entre le regard et le psychisme autour de l'axe " œil-cerveau ». D'autre part, nous insisterons sur le caractère problématique, incertain et ambigu de ce processus d'élaboration conceptuelle et fictionnelle qui est au cœur de la création kubrickienne et qui doit avant tout être entendu, non pas comme un geste de clôture du sens, mais comme un mouvement indéfini de construction et de déconstruction.

\title{
Pierre Beylot
}

Université Bordeaux Montaigne Pierre.Beylot@u-bordeaux-montaigne.fr

\section{Résumé}

Quel rapport le cinéma entretient-il avec la pensée ? C'est au travers de la métaphore du « filmcerveau " proposée par Gilles Deleuze que nous envisageons cette question sous trois aspects : celui de la pensée en acte du cinéaste qui déploie une armature conceptuelle qui enveloppe et circonscrit le développement du récit sans que, cependant, celui-ci lui soit totalement inféodé ; le deuxième aspect est celui du monde fictionnel envisagé comme monde-cerveau peuplé de figures - monolithe, labyrinthe, huis clos... - où se cristallise la relation entre espace mental et espace physique ; enfin, la corporéité du film-cerveau s'affirme dans la relation entre l'œil et le cerveau quand le cinéma de Kubrick donne à voir des univers mentaux obsessionnels et traumatiques et souligne en même temps les limites de cette exploration psychique. Ce parcours dans le labyrinthe du film-cerveau sera mené au travers d'une période particulièrement dense de la création kubrickienne, allant de 2001 (1968) à Shining (1980).

\section{Mots-clés}

"Film-cerveau ", cinéma et espace mental, cinéma et abstraction, motif de l'œil au cinéma, exosquelette narratif.

\begin{abstract}
What kind of connection does cinema entertain with thought? Through Gilles Deleuze's metaphor of a "film-brain", I envision this question under three different lights: the active thought of the filmmaker which builds up a conceptual framework, envelopping and circumscribing the narrative yet without fully subordinating it; secondly, the fictive world envisionned as world-brain and filled with motifs - monolith, maze, enclosed space - where the relation between mental space and physical space crystallises; lastly, the corporeality of the film-brain which is highlighted by the eye-brain relationship whenever Kubrick's cinema depicts obsessive, traumatic mental spaces, and which simultaneously underlines the limits of such mental exploration. This journey through the maze of the film-brain relies on Kubrick's particularly active creative period, from 2001 (1968) up to The Shining (1980).

\section{Keywords}

"film-brain", cinema and mental space, cinema and abstraction, motif of the eye on film, narrative framework.
\end{abstract}

la puissance commune de ce qui force à penser et de ce qui pense sous le choc : un noochoc ", autrement dit le « choc qui éveille le penseur en vous ", L'Image-Temps, op. cit., p. 204. 


\section{NOTICE BIOGRAPHIQUE}

Pierre Beylot, professeur des universités en Études cinématographiques et audiovisuelles à l'Université Bordeaux Montaigne, est spécialiste du récit de fiction au cinéma et à la télévision. Il est notamment l'auteur du Récit audiovisuel (Armand Colin, 2005), d'Analyse d'une cuvre: Conte d'été, É. Rohmer, 1996 (avec Martin Barnier, Vrin, 2011), de Buffet froid de Bertrand Blier (Atlande, 2017). Il a également dirigé ou co-dirigé plusieurs ouvrages collectifs, parmi lesquels Emprunts et citations dans le champ artistique (2004), Les Séries policières (co-dir., 2004), Fictions patrimoniales sur grand et petit écran. Contours et enjeux d'un genre intermédiatique (co-dir., 2009), Les Images en question. Cinéma, télévision, nouvelles images: les voies de la recherche (co-dir., 2011), Images pour/suite : remake, franchise, filiation (co-dir.), $M A P, \mathrm{n}^{\circ} 10,2018$. Il est actuellement responsable de la section Cinéma et du Master Cinéma et Audiovisuel de l'Université Bordeaux Montaigne. 

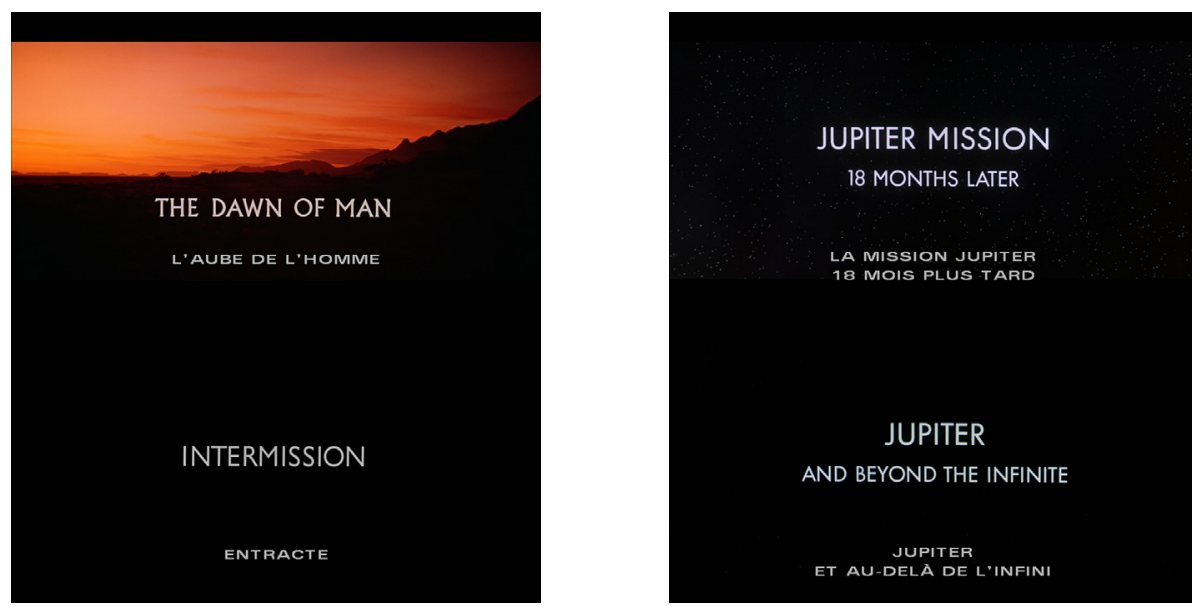

Figure 1 : 2001 : l'odyssée de l'espace.
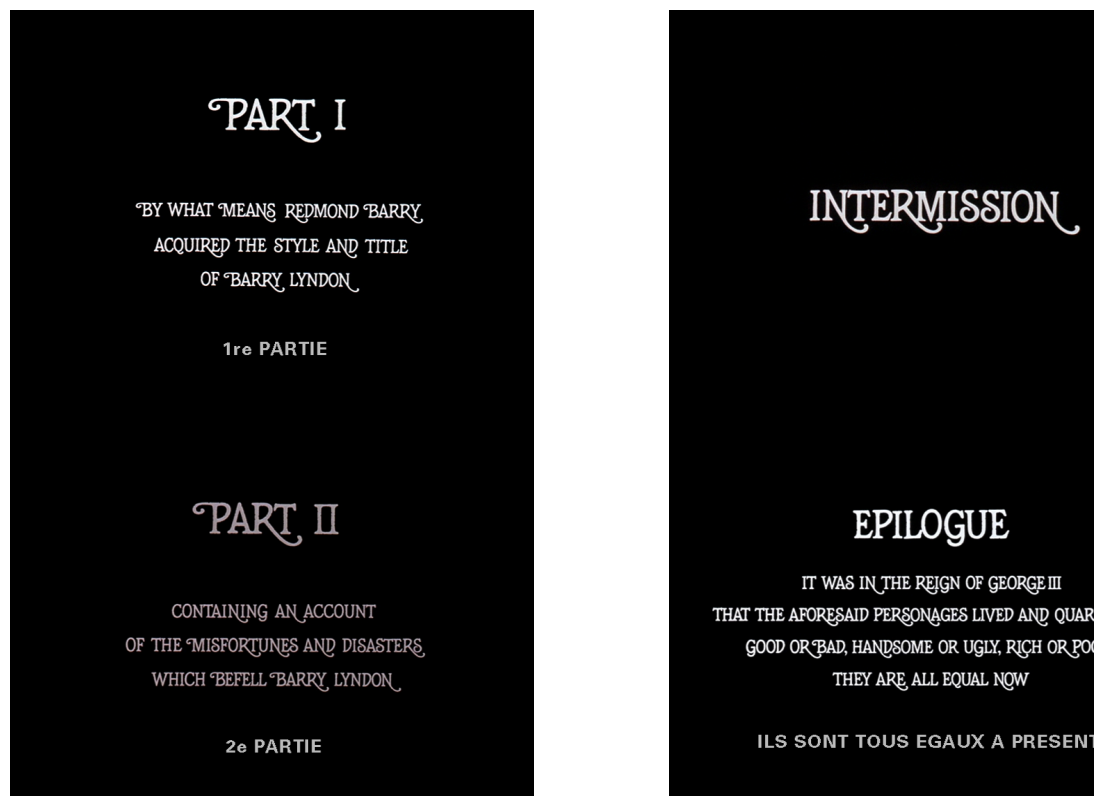

W WAT MEANE REDMOND BARR

INTERMISSION OF 'BARRY LYNDON

Figure 2 : Barry Lyndon.

\section{EPILOGUE}

IT WAS INTHE REIGN OF GEORGEIII THAT THE AFORESAID PERSONAGGES LIVED AND QUARRELED; GOOD ORBBAD, HANDSOME OR UGLY, RICH OR POOR, THEY ARE ALL EQUAL NOW

ILS SONT TOUS EGAUX A PRESENT. 


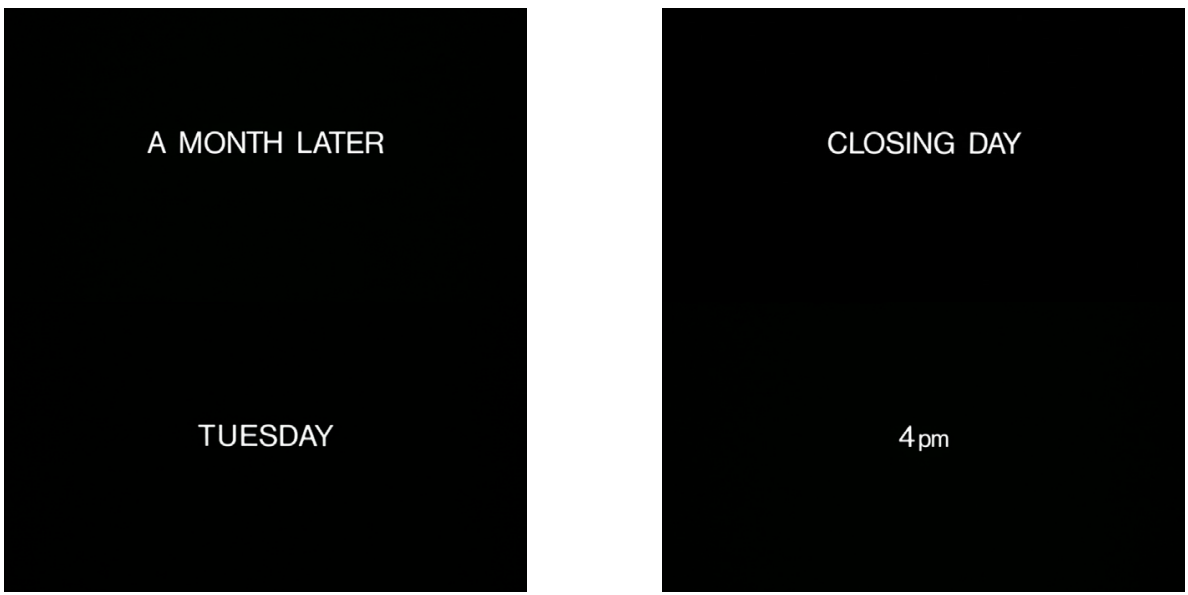

Figure 3 : Shining.
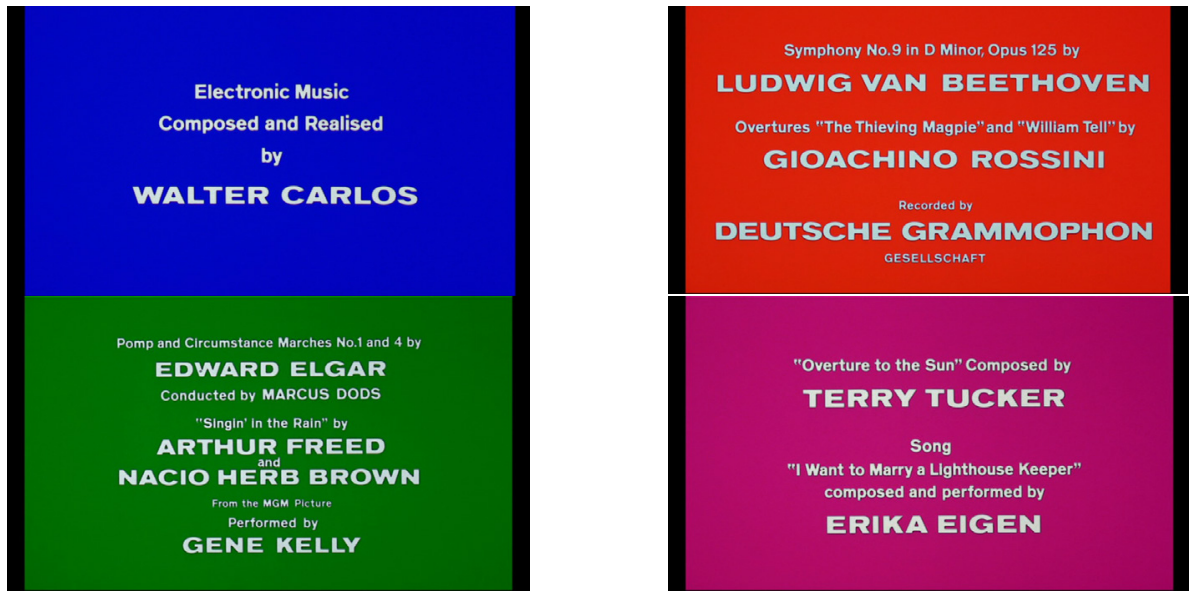

Figure 4 : Orange mécanique. 


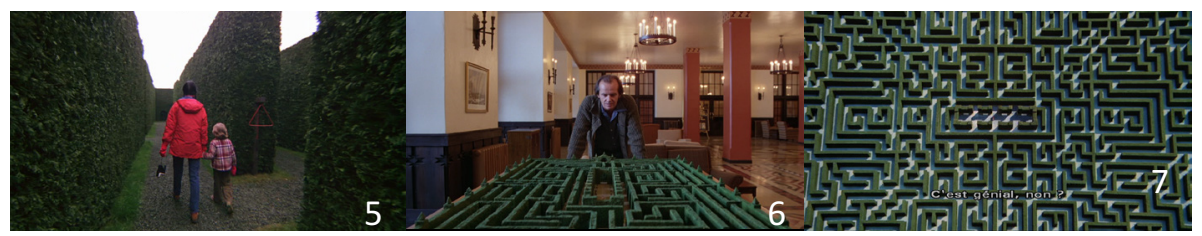

Figures 5, 6 et 7 : Shining.

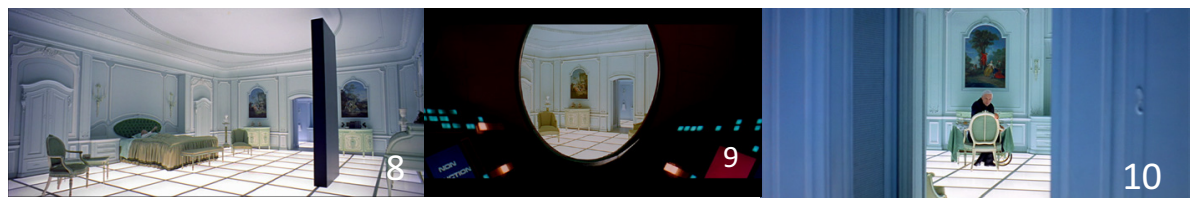

Figures 8, 9 et $10: 2001$.

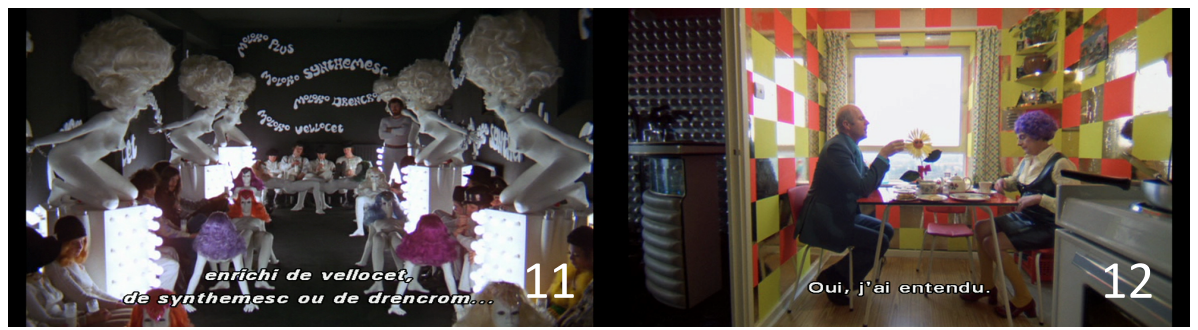

Figures 11 et 12 : Orange mécanique.

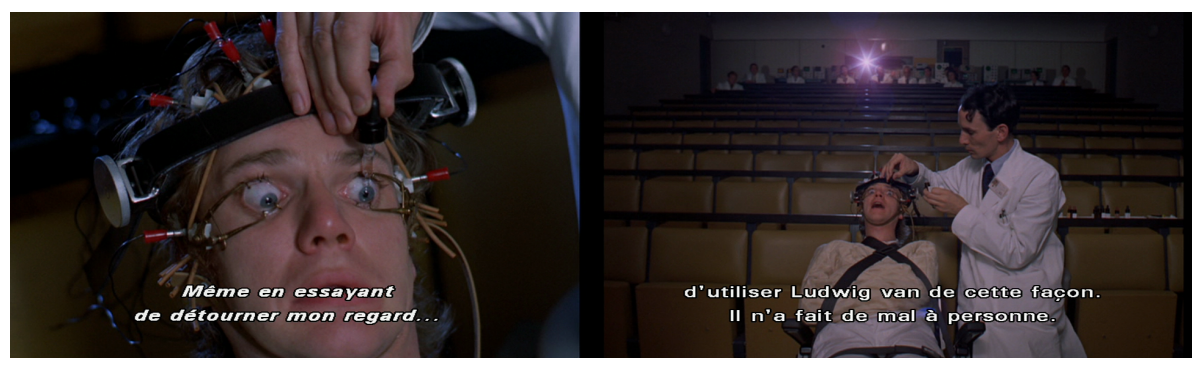

Figure 13 : Orange mécanique. 


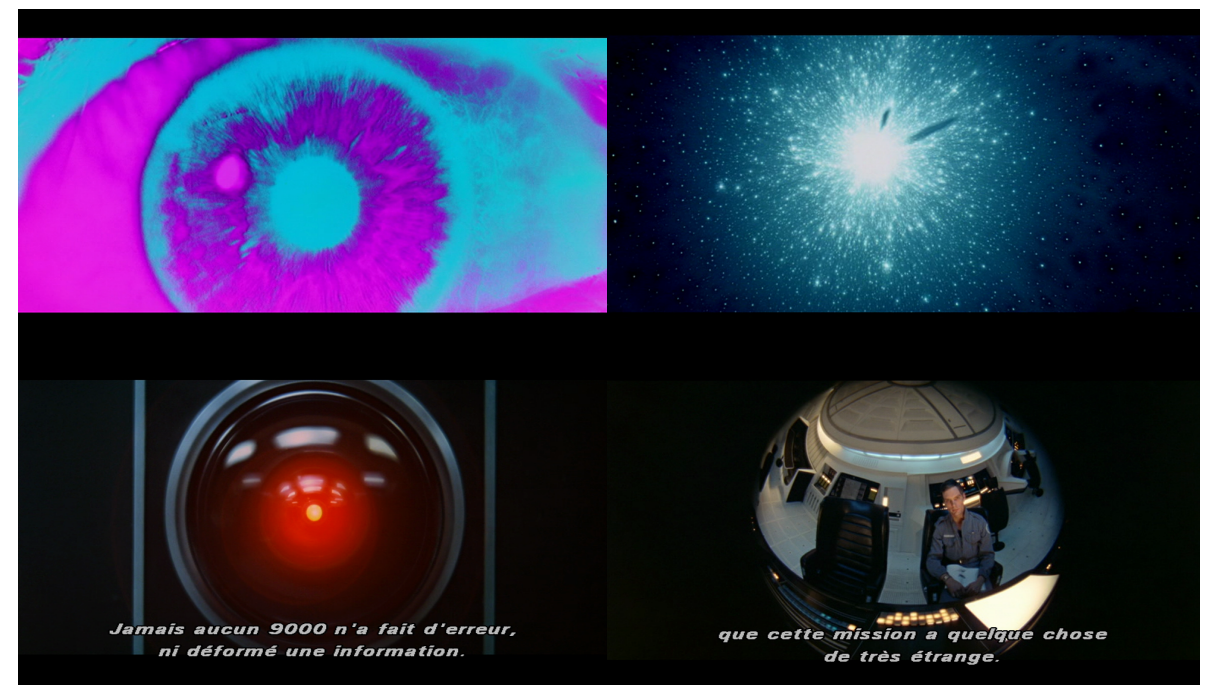

Figure 14: 2001.

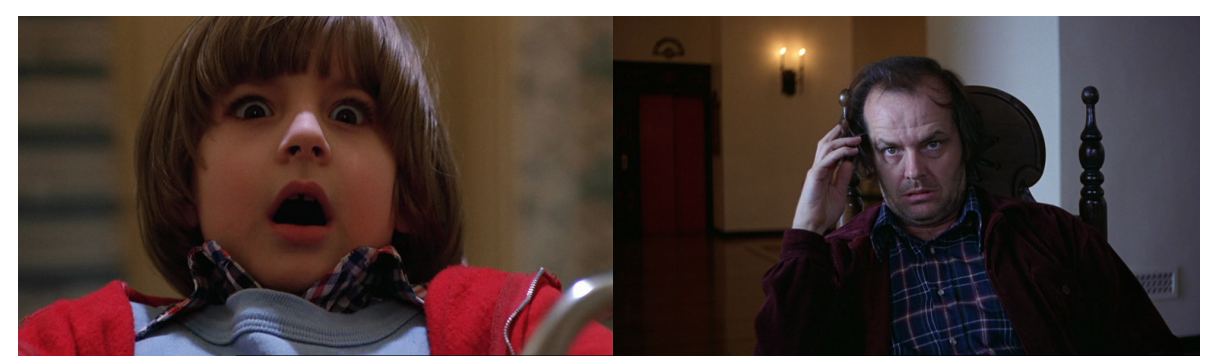

Figure 15: 2001 . 